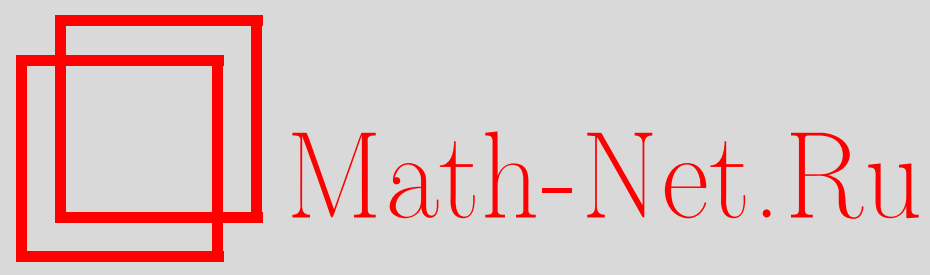

И. Р. Каюмов, Точные оценки интегральных средних для трех классов областей, Матем. заметки, 2004, том 76, выпуск 4, 510-516

DOI: https://doi.org/10.4213/mzm127

Использование Общероссийского математического портала Math-Net.Ru подразумевает, что вы прочитали и согласны с пользовательским соглашением http://www.mathnet.ru/rus/agreement

Параметры загрузки:

IP: 3.95 .254 .165

26 апреля 2023 г., $15: 17: 32$

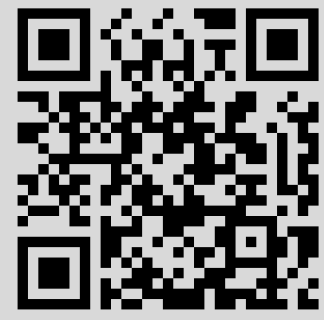




\section{ТОЧНЫЕ ОЦЕНКИ ИНТЕГРАЛЬНЫХ СРЕДНИХ ДЛЯ ТРЕХ КЛАССОВ ОБЛАСТЕЙ}

\section{И.Р. Каюмов}

В работе доказана точная оценка

$$
\int_{0}^{2 \pi}\left|F^{\prime}\left(e^{i \theta}\right)\right|^{p} d \theta \leqslant \sqrt{\pi} 2^{1+p} \frac{\Gamma(1 / 2+p / 2)}{\Gamma(1+p / 2)}, \quad p>-1,
$$

где $\boldsymbol{F}$ - конформное отображение области $D^{-}=\{\zeta:|\zeta|>1\}$ на внешность выпуклой кривой, $F^{\prime}(\infty)=1$. При $p=1$ этот результат принадлежит Полиа и Шифферу. Получены также несколько обобщений этой оценки при других геометрических предположениях о строении области $F\left(D^{-}\right)$.

Библиография: 15 названий.

Как обычно, обозначим через $S$ класс аналитических и однолистных в круге $D=$ $\{|z|<1\}$ функций $f$ с нормировкой $f(0)=f^{\prime}(0)-1=0$, а через $\Sigma$ - класс аналитических и однолистных во внешности единичного круга $D^{-}=\{|\zeta|>1\}$ функций $F$ с разложением

$$
F(\zeta)=\zeta+a_{0}+\frac{a_{1}}{\zeta}+\cdots
$$

в окрестности точки $\zeta=\infty$. Через $K$ обозначим подкласс функций класса $S$, которые отображают круг на почти-выпуклую область в смысле Каплана [1].

Используя известные результаты Бернштейна [2], Ленг [3] доказал следующее утверждение, которое является обобщением результатов, полученных Клуни, Дюреном и МакГрегором [4], [5].

Пусть $\Phi-$ вьпуклая неубывающая функиия на $(-\infty,+\infty)$. Тогда для любой $f \in K$ июбых $0<r<1$ выполнено следующее неравенство:

$$
\int_{0}^{2 \pi} \Phi\left(\log \left|f^{\prime}\left(r e^{i \theta}\right)\right|\right) d \theta \leqslant \int_{0}^{2 \pi} \Phi\left(\log \left|k^{\prime}\left(r e^{i \theta}\right)\right|\right) d \theta
$$

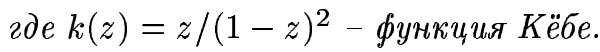

Работа выполнена при поддержке Российского фонда фундаментальных исследований, грант № 02-01-00168. 
В классе $\Sigma$ аналогичные вопросы оказались более сложными. А именно, не установлен даже точньй порядок роста при $R \rightarrow 1$ величины

$$
\int_{|\zeta|=R}\left|F^{\prime}(\zeta)\right|^{p} d \theta, \quad-2<p<2 .
$$

Как показали Макаров [6], Карлесон и Джонс [7], сложность этой проблемы связана с тем, что граница области $F\left(D^{-}\right)$для экстремальной функции должна иметь фрактальную структуру.

Лучшие верхние оценки этой величины принадлежали Поммеренке [8]. В 1999 г. Бертильсону [9] удалось их слегка улучшить. Лучшие нижние оценки при $|p|<1$ получены в работах автора [10], [11] (в этих работах даются оценки снизу для ограниченных однолистных функций, что влечет нижние оценки для функций класса $\Sigma$ ).

В данной работе мы докажем аналоги теоремы Ленга для трех подклассов однолистных функций.

Пусть $Г$ - выпуклая кривая на плоскости. Предположим, что размер кривой фиксирован условием на внешний конформный радиус: $\rho\left(\infty, D_{\Gamma}\right)=\rho$, где $D_{\Gamma}-$ внешность $\Gamma$. Множество всех таких кривьх обозначим через $C_{\rho}=\{\Gamma\}$.

В известной монографии Полиа и Сегё [12, с. 38] была поставлена следующая проблема: найти точную оценку длины выпуклой кривой через внешний конформньй радиус $\rho$. Полиа и Шиффер [13] доказали следующее утверждение.

Для любой $\Gamma \in C_{\rho}$ имеют место точные оченки

$$
2 \pi \rho \leqslant l(\Gamma) \leqslant 8 \rho .
$$

Здесь $l(\Gamma)-$ длина кривой $Г$.

Отметим, что оценка снизу $l(\Gamma) \geqslant 2 \pi \rho$ доказьвается просто, и равенство имеет место лишь для окружности. Доказательство правого неравенства из (1) было проведено в [2] с помощью вариационного метода для многоугольников. Случай произвольных вьпуклых областей получался путем предельного перехода. В данной работе другим методом мы докажем более обший результат, которьй содержит в себе результат Полиа и Шифофера как частньй случай.

Через $\Sigma_{0}$ обозначим класс, содержащий функции из $\Sigma$, которые отображают внешность круга на внешность вьпуклого множества.

Теперь сформулируем наш основной результат.

ТЕОрема 1. Пусть $F \in \Sigma_{0} u \Psi(z)-$ произвольная аналитическая в круге $\{|z-1|$ $<1\}$ функция. Тогда для любого $R>1$ выполнено следующее неравенство:

$$
\int_{|\zeta|=R}\left|\Psi\left(F^{\prime}(\zeta)\right)\right|^{2} d \theta \leqslant \int_{|\zeta|=R}\left|\Psi\left(1+\frac{1}{\zeta^{2}}\right)\right|^{2} d \theta, \quad \zeta=R e^{i \theta} .
$$

В частности, при $p>-1$ для любой функиии $F \in \Sigma_{0}$ имеет место точная оченка

$$
\int_{0}^{2 \pi}\left|F^{\prime}\left(e^{i \theta}\right)\right|^{p} d \theta \leqslant \sqrt{\pi} 2^{1+p} \frac{\Gamma(1 / 2+p / 2)}{\Gamma(1+p / 2)} .
$$

В обоих неравенствах равенство достигается, например, для функиии Жуковского $F(\zeta)=\zeta-1 / \zeta$. 
ДокАЗАТЕЛЬСТво. В отличие от Ленга, мы не пользуемся симметризацией, а опираемся на следующий принцип мажорации для аналитических функций. А именно, рассмотрим функции $f(z)$ и $g(z)$, аналитичные в круге $D$, причем $f(0)=g(0)$. Функция $f(z)$ назьвается подчиненной функции $g(z)$, если $f(z)=g(\varphi(z))$, где $\varphi(z)$ аналитична в $D$, $|\varphi|<1$ в $D$ и $\varphi(0)=0$ (см. [14, с. 356]). Справедливо следующее утверждение, принадлежащее Литтлвуду.

ЛЕмма 1 [14, с. 357]. Пусть $f$ подчинена $g$ и $f(0)=0$. Тогда для любого $0<r<1$

$$
\int_{0}^{2 \pi}\left|f\left(r e^{i \theta}\right)\right|^{2} d \theta \leqslant \int_{0}^{2 \pi}\left|g\left(r e^{i \theta}\right)\right|^{2} d \theta
$$

Известно, что для функций класса $\Sigma_{0}$ справедливо неравенство Голузина (см. напримep [1]):

$$
\left|F^{\prime}-1\right|<1
$$

что влечет следующее представление

$$
F^{\prime}(\zeta)=1+\phi(\zeta), \quad \zeta \in D^{-}
$$

где функция $\phi$ обладает свойствами: $|\phi(\zeta)|<1$ для $\zeta \in D^{-}$и в окрестности точки $\zeta=\infty$ имеет разложение $\phi(\zeta)=d_{2} / \zeta^{2}+d_{3} / \zeta^{3}+\cdots$.

Переходя к кругу $D$ с помощью замены $\zeta=1 / z$, имеем $F^{\prime}(1 / z)=1+\psi(z)$, где $\psi(z)=d_{2} z^{2}+h_{3} z^{3}+\cdots-$ аналитическая в круге $D$ функция, область значений которой лежит также в $D$.

Введем функцию $g(z)=\Psi(1+\psi(z / R))-\Psi(1)$, которая, очевидно, подчинена функции $\Psi\left(1+z / R^{2}\right)-\Psi(1)$ в силу леммы Шварца [14, с. 319]. Без ограничения общности можно считать, что $\Psi(1)$ является вещественным числом.

Применяя к $g$ лемму 1 и переходя к пределу при $r \rightarrow 1$, получаем

$$
\int_{0}^{2 \pi}\left|g\left(e^{i \theta}\right)\right|^{2} d \theta \leqslant \int_{0}^{2 \pi}\left|\Psi\left(1+\frac{e^{i \theta}}{R^{2}}\right)-\Psi(1)\right|^{2} d \theta
$$

что эквивалентно неравенству

$$
\begin{gathered}
\int_{0}^{2 \pi}\left|\Psi\left(F^{\prime}\left(R e^{-i \theta}\right)\right)\right|^{2} d \theta+2 \Psi(1) \int_{0}^{2 \pi} \operatorname{Re} \Psi\left(F^{\prime}\left(R e^{-i \theta}\right)\right) d \theta+2 \pi \Psi(1)^{2} \\
\leqslant \int_{0}^{2 \pi}\left|\Psi\left(1+\frac{e^{i \theta}}{R^{2}}\right)\right|^{2} d \theta+2 \Psi(1) \int_{0}^{2 \pi} \operatorname{Re} \Psi\left(1+\frac{e^{i \theta}}{R^{2}}\right) d \theta+2 \pi \Psi(1)^{2} .
\end{gathered}
$$

По теореме о среднем для гармонических функций вторые слагаемые в левой и правой частях последнего неравенства совпадают. Поэтому получаем

$$
\int_{0}^{2 \pi}\left|\Psi\left(F^{\prime}\left(R e^{-i \theta}\right)\right)\right|^{2} d \theta \leqslant \int_{0}^{2 \pi}\left|\Psi\left(1+\frac{e^{i \theta}}{R^{2}}\right)\right|^{2} d \theta
$$


Отсюда следует доказьваемое неравенство

$$
\int_{|\zeta|=R}\left|\Psi\left(F^{\prime}(\zeta)\right)\right|^{2} d \theta \leqslant \int_{|\zeta|=R}\left|\Psi\left(1+\frac{1}{\zeta^{2}}\right)\right|^{2} d \theta
$$

с учетом следующего соотношения, легко проверяемого на основании равенства Парсеваля:

$$
\int_{0}^{2 \pi}\left|\Psi\left(1+\frac{e^{i \theta}}{R^{2}}\right)\right|^{2} d \theta=\int_{|\zeta|=R}\left|\Psi\left(1+\frac{1}{\zeta^{2}}\right)\right|^{2} d \theta
$$

Заметим, что непосредственное применение леммы Литтлвуда к функции $\Psi\left(F^{\prime}(1 / z)\right)$ дало бы лишь неточную оценку.

Для доказательства второго утверждения теоремы 1 положим $\Psi_{p}(z)=z^{p / 2}$, где ветвь фиксирована условием $\Psi_{p}(1)=1$. Применяя первую часть теоремы 1 и переходя к пределу при $R \rightarrow 1$ на основании теоремы Рисса [14, с. 390], получаем

$$
\int_{0}^{2 \pi}\left|F^{\prime}\left(e^{i \theta}\right)\right|^{p} d \theta \leqslant 2^{2+p} \int_{0}^{\pi / 2} \cos ^{p} \theta d \theta=2^{1+p} B\left(\frac{p+1}{2}, \frac{1}{2}\right)
$$

где $B$ - бета-функция Эйлера. Доказательство легко завершается теперь применением известного соотношения между гамма- и бета-функциями.

Полагая $p=1$, получаем результат Полиа и Шиффера.

При $R>\sqrt{2}+1$ теорему 1 можно усилить следующим образом. Обозначим через $\Sigma^{*} \subset \Sigma$ класс звездных функций, т.е. функций, которые отображают круг на внешность звездного множества. Ясно, что $\Sigma_{0} \subset \Sigma^{*}$. Имеет место следующая

Теорема 2. Пусть $F=\zeta+\sum_{k=1}^{\infty} a_{k} \zeta^{-k} \in \Sigma^{*} u \Psi(z)-$ произвольная аналитическая в круге $\{|z-1|<3-2 \sqrt{2}\}$ функиия. Тогда для любого $R \geqslant \sqrt{2}+1$ выполнено следующее неравенство:

$$
\int_{|\zeta|=R}\left|\Psi\left(F^{\prime}(\zeta)\right)\right|^{2} d \theta \leqslant \int_{|\zeta|=R}\left|\Psi\left(1+\frac{1}{\zeta^{2}}\right)\right|^{2} d \theta, \quad \zeta=R e^{i \theta}
$$

ДокАЗАТЕЛЬСТВо такое же, как и у теоремы 1 , поскольку для функций класса $\Sigma^{*}$ при $|\zeta| \geqslant \sqrt{2}+1$ вьполнено неравенство [14, с. 514]

$$
\left|F^{\prime}(\zeta)-1\right| \leqslant \frac{1}{|\zeta|^{2}}
$$

Заметим также, что без дополнительных предположений на функцию $\Psi$ или $F$, число $\sqrt{2}+1$ не может быть заменено на меньшее. Это следует из точности оценки Голузина (см. $[14$, c. 514]). 
Теперь перейдем к рассмотрению аналогичных вопросов в более широких классах однолистных функций. Бертильсон [9, с. 111] показал, что для достаточно малых $r$, зависяших от $p$, вьполнено неравенство

$$
\int_{|z|=r}\left|f^{\prime}(z)\right|^{p} d \theta \leqslant \int_{|z|=r}\left|k^{\prime}(z)\right|^{p} d \theta
$$

для всех $f \in S$ при условии, что $C_{n} \neq 0$ для каждого $n \geqslant 0$, где $C_{n}-$ коэффициенты ряда Тейлора для функции $1 / k^{\prime}(z)^{p}$.

Мы рассмотрим более общий вопрос для функций класса $S_{R}$, которьй состоит из функций класса $S$, тейлоровские коэффициенты которых вешественны.

Имеет место

ТЕОРема 3. Пусть функиия $\Phi$ аналитична в окрестности 1 и $\Phi(1) \neq 0$. Тогда найдется положительное число $r_{\Phi}$ такое, что

$$
\sup _{f \in S_{R}} \int_{|z|=r}\left|\Phi\left(f^{\prime}(z)\right)\right| d \theta=\int_{|z|=r}\left|\Phi\left(k_{ \pm}^{\prime}(z)\right)\right| d \theta
$$

для любого $r \leqslant r_{\Phi}$, где $k_{ \pm}(z)=z /(1 \pm z)^{2}$.

ДокАЗАТЕЛЬСТВо. Без ограничения общности можно считать, что $\Phi(z)=\Psi^{2}(z)$. Далее вводя новую функцию $F(z)=\Psi(z)-\Psi(1)$, имеем

$$
\int_{|z|=r}\left|\Psi\left(f^{\prime}(z)\right)\right|^{2} d \theta=\int_{|z|=r}\left|F\left(f^{\prime}(z)\right)\right|^{2} d \theta+2 \pi|\Psi(1)|^{2} .
$$

Таким образом, нам нужно доказать, что

$$
\sup _{f \in S_{R}} \int_{|z|=r}\left|F\left(f^{\prime}(z)\right)\right|^{2} d \theta \leqslant \int_{|z|=r}\left|F\left(k_{ \pm}^{\prime}(z)\right)\right|^{2} d \theta
$$

где функция $F$ аналитична в окрестности 1 и $F(1)=0$. Заметим, что последнее условие в силу известной леммы Шварца влечет существование абсолютных положительных констант $C_{1}$ и $r_{1}$ таких, что для любой функции $f \in S_{R}$ вьполнено неравенство

$$
\left|F\left(f^{\prime}(z)\right)\right| \leqslant C_{1} r^{n}, \quad r<r_{1}
$$

где $n$ - наименьшее натуральное число такое, что $F^{(n)}(1) \neq 0$.

Пусть $f \in S_{R}$. Тогда $f$ представима в виде [14]

$$
f(z)=\frac{z}{1-z^{2}} \frac{1+\varphi(z)}{1-\varphi(z)}
$$

где $\varphi$ является аналитической в круге $D$ функцией и $|\varphi(z)| \leqslant|z|, z \in D$. Без ограничения общности будем считать, что $\varphi^{\prime}(0)=a \geqslant 0$. Известное неравенство Бибербаха 
влечет оценку для $a: 0 \leqslant a \leqslant 1$. Введем функцию $g_{a}(z)=z /(1-a z)^{2}$ и рассмотрим разность

$$
\begin{aligned}
\left|f(z)-g_{a}(z)\right| & =|z|\left|\frac{1}{1-z^{2}} \frac{1+\varphi}{1-\varphi}-\frac{1}{1-z^{2}} \frac{1+a z}{1-a z}+\frac{z^{2}\left(1-a^{2}\right)}{\left(1-z^{2}\right)(1-a z)^{2}}\right| \\
& \leqslant \frac{2|z||\varphi-a z|}{\left|1-z^{2}\right||1-\varphi(z)||1-a z|}+\frac{|z|^{3}\left(1-a^{2}\right)}{\left|1-z^{2}\right||1-a z|^{2}}
\end{aligned}
$$

Рассмотрим, теперь, разность

$$
|\varphi(z)-a z|=\left|\sum_{k=2}^{\infty} a_{k} z^{k}\right|
$$

Известно [14], что $\left|a_{k}\right| \leqslant\left(1-a^{2}\right)$. Отсюда, $|\varphi-a z| \leqslant\left(1-a^{2}\right) r^{2} /(1-r)^{2}$. Следовательно, найдутся положительные постоянные $C_{2}, r_{2}$ такие, что

$$
\left|f(z)-g_{a}(z)\right| \leqslant C_{2}\left(1-a^{2}\right) r^{3}, \quad r \leqslant r_{2}
$$

Полученное неравенство влечет существование положительных констант $C_{3}, r_{3}$ таких, что

$$
\left|f^{\prime}(z)-g_{a}^{\prime}(z)\right| \leqslant C_{3}\left(1-a^{2}\right) r^{2}, \quad r \leqslant r_{3}
$$

Отсюда следует, что существуют положительные $C_{4}, r_{4}$ такие, что

$$
\left|F\left(f^{\prime}(z)\right)-F\left(g_{a}^{\prime}(z)\right)\right| \leqslant C_{4}\left(1-a^{2}\right) r^{n+1}, \quad r \leqslant r_{4}
$$

Таким образом, имеем

$$
\begin{aligned}
& \int_{|z|=r}\left|F\left(f^{\prime}(z)\right)\right|^{2} d \theta \leqslant \int_{|z|=r}\left|F\left(f^{\prime}(z)\right)-F\left(g_{a}^{\prime}(z)\right)\right|^{2} d \theta \\
& \quad+2 \int_{|z|=r}\left|F\left(f^{\prime}(z)\right)-F\left(g_{a}^{\prime}(z)\right)\right|\left|F\left(g_{a}^{\prime}(z)\right)\right| d \theta+\int_{|z|=r}\left|F\left(g_{a}^{\prime}(z)\right)\right|^{2} d \theta .
\end{aligned}
$$

Теперь, применяя неравенства (2) и (3), получаем неравенство

$$
\begin{aligned}
\int_{|z|=r}\left|F\left(f^{\prime}(z)\right)\right|^{2} d \theta \leqslant & C_{4}^{2} r^{2 n+2}\left(1-a^{2}\right)^{2}+2 C_{4}\left(1-a^{2}\right) C_{1} r^{2 n+1} \\
& +\left|\frac{F^{(n)}(1)}{n !}\right|^{2}(2 a r)^{2 n}+\sum_{k=n+1}^{\infty}\left|b_{k}\right|^{2}(r a)^{2 k}
\end{aligned}
$$

где

$$
\int_{|z|=r}\left|F\left(g_{a}^{\prime}(z)\right)\right|^{2} d \theta=\left|\frac{F^{(n)}(1)}{n !}\right|^{2}(2 a r)^{2 n}+\sum_{k=2}^{\infty}\left|b_{k}\right|^{2}(r a)^{2 k}
$$


Используя стандартные методы геометрической теории функций комплексного переменного, нетрудно убедиться, что $\left|b_{k}\right| \leqslant C_{5} k^{2}$, где $C_{5}$ - абсолютная константа.

Максимизируя правую часть в (4) по параметру $a$, приходим к выводу, что при достаточно малых $r$ максимум достигается в точке $a=1$ (для этого достаточно убедиться, что в точках локальных экстремумов эта функция ведет себя как $O\left(r^{2 n+n /(n-1)}\right)$ при малых $r$ ).

Интересно отметить, что $r_{\Phi}$ может быть строго меньше 1 , даже в случае, когда $\Phi$ аналитична во всей плоскости за исключением 0 , т.е. на всей области значений производных функций из класса $S$. Из результатов Карлесона и Макарова [15] следует, что в случае, когда $\Phi(z)=z^{p},-2<p<1 / 3$, вьполнена оценка $r_{\Phi}<1$. Заметим также, что в [10] установлено, что константу $1 / 3$ можно увеличить до 0.34 .

В заключение хочу выразить благодарность Ф. Г. Авхадиеву за полезные замечания и внимание к работе.

\section{СПИСОК ЦИТИРОВАННОЙ ЛИТЕРАТУРЫ}

[1] Авхадиев Ф.Г., Аксентьев Л.А. Основные результаты в достаточных условиях однолистности аналитических функций // УМН. 1975. Т. 30. №4(184). С. 3-60.

[2] Baernstein A. Integral means, univalent functions and circular symmetrization // Acta Math. 1974. V. 133. P. 139-169.

[3] Leung Y.J. Integral means of the derivatives of some univalent functions // Bull. London Math. Soc. 1979. V. 11. P. 289-294.

[4] Clunie J., Duren P. L. Addendum: An arclength problem for close-to-convex functions // J. London Math. Soc. 1966. V. 41. P. 181-182.

[5] MacGregor T.H. Applications of extreme point theory to univalent functions // Michigan Math. J. 1972. V. 19. P. 361-376.

[6] Makarov N. G. Fine structure of harmonic measure // St. -Petersbg. Math. J. 1999. V. 10. № 2. P. 217-268.

[7] Carleson L., Jones P.W. On coefficient problems for univalent functions and conformal dimension // Duke Math. J. 1992. V. 66. № 2. P. 169-206.

[8] Pommerenke Ch. Boundary Behaviour of Conformal Maps. Berlin: Springer-Verlag, 1992.

[9] Bertillson D. On Brennan's Conjecture in Conformal Mapping. Doctoral Thesis. Stockholm: Royal Institute Technology, 1999.

[10] Kayumov I. R. Lower estimates for the integral means spectrum // Complex Variables. 2001. V. 44. P. $165-171$.

[11] Kayumov I. R. Lower estimate for the integral means spectrum for $p=-1 / /$ Proc. Amer. Math. Soc. 2001. V. 130. № 4. P. 1005-1007.

[12] Полиа Г., Сегё Г. Изопериметрические неравенства математической физики. М.: Физматлит, 1962.

[13] Pólya G., Shiffer M. Sur la reprépresentation conforme de l'extérieur d'une courbe fermée convex // C. R. Acad. Sci. Paris. 1959. V. 248. № 20. P. 2837-2839.

[14] Голузин Г. М. Геометрическая теория функций комплексного переменного. М.: Наука, 1966.

[15] Carleson L., Makarov N. G. Some results connected with Brennan's conjecture // Ark. Mat. 1994. V. 32. P. 33-62.

НИИ математики и механики им. Н. Г. Чеботарева

Поступило при Казанском государственном университете 26.12 .2002

E-mail: ikayumov@ksu.ru 